\title{
Monitoramento externo da qualidade dos exames citopatológicos cervicais realizados pelo Sistema Único de Saúde (SUS) no Estado de Mato Grosso do Sul
}

\author{
External quality control for cervical cytology exams performed in the \\ Brazilian Public Health System of Mato Grosso do Sul State
}

Artigo Original

Palavras-chave

Controle de qualidade

Citodiagnóstico

Esfregaço vaginal

Neoplasias do colo do útero

Keywords

Quality control Cytodiagnosis Vaginal smears

Uterine cervical neoplasms

\section{Resumo}

OBJETIVO: Analisar a concordância de diagnósticos citopatológicos de laboratórios credenciados e os verificados no monitoramento. MÉTODOS: Foram calculados o coeficiente de concordância global e o coeficiente Kappa de Cohen de uma amostra de conveniência dos exames selecionados mensalmente pelo Sistema de Informação do Câncer do Colo do Útero (SISCOLO) para monitoramento externo da qualidade (MEQ) dos 15 laboratórios que realizaram exames citopatológicos para o SUS entre 2002 e 2011 no Estado do Mato Grosso do Sul (região central do Brasill. A comparação entre os valores de concordância (coeficiente de concordância global e coeficiente Kappa) dos anos inicial e final foi computada por meio da variação absoluta (delta) e variação percentual relativa (VPR). RESULTADOS: Foram encaminhadas para releitura 15.989 esfregaços, dos quais 48, 1\% apresentavam laudo normal/alteração benigna, seguidos de atipia/lesão de baixo grau (36,3\%), lesão de alto grau/carcinoma/adenocarcinoma (4,2\%) e insatisfatórios para leitura $(11,4 \%)$. O coeficiente de concordância global oscilou entre 0,2 e 1,0, sendo que o valor mediano passou de 0,7 em 2002 para 1,0 em 2011 (VPR=+36,6\%). Nesse mesmo período, os valores medianos do coeficiente Kappa aumentaram de 0,5 para 0,9 (VPR=+80,8\%). CONCLUSÕES: Conclui-se pela exequibilidade do $M E Q$ dos exames citopatológicos na esfera estadual e que sua implementação resulta em melhoria nos diagnósticos realizados na rede SUS.

\section{Abstract}

PURPOSE: To evaluate the diagnostic agreement between the cytopathology reports issued by accredited laboratories and those obtained by quality control. METHODS: We calculated the overall agreement and Cohen's kappa coefficients of a convenience sample of smears selected monthly by the Information System of Cervical Cancer (SISCOLO) for External Quality Control of the 15 laboratories that performed cytopathological PAP tests for the Brazilian Public Health System (SUS) between 2002 and 2011 in Mato Grosso do Sul, a State of the Midwest Region of the country. A comparison of the reliability values (coefficient of concordance and Kappa coefficient) between the initial and final years was computed by the absolute change (delta) and relative percent difference (RPD). RESULTS: There were 15.989 smears sent for rereading, $48.1 \%$ of which had a report of normal/benign changes, followed by atypical/low-grade squamous intraepithelial lesions (36.3\%), high grade squamous intraepithelial lesion/carcinoma/adenocarcinoma $(4.2 \%)$, and unsatisfactory (1 $1.4 \%)$. The overall correlation coefficient ranged between 0.2 and 1.0 , and the median value increased from 0.7 in 2002 to 1 in 2011 (RPD=+36.6\%). During the same period, the median values of the Kappa coefficient increased from 0.5 to 0.9 (RPD $=+80.8 \%$ ). CONCLUSIONS: These results emphasize the feasibility of the External Quality Control of cytopathology at the state level and its implementation results in improvement in the diagnoses performed in the SUS network.
Correspondêncio

Hilda Guimarães de Freitas Parque dos Poderes, Bloco 7 (EP: 79031-902

Campo Grande (MS), Brasil

Recebido

02/07/2012
Trabalho realizado na Secretaria Estadual de Saúde de Mato Grosso do Sul - Cuiabá (MT), Brasil.

'Secretaria Estadual de Saúde de Mato Grosso do Sul - Cuiabá (MT), Brasil.

${ }^{2}$ Instituto Nacional de Câncer - INCA; Universidade Federal do Estado do Rio de Janeiro - UNIRIO - Rio de Janeiro (RJ), Brasil. 


\section{Introdução}

Em 2012, de acordo com as estimativas do Instituto Nacional de Câncer ${ }^{1}$ (INCA), o câncer do colo do útero ocupará a segunda posição entre as localizações de câncer com maior incidência entre as mulheres brasileiras (17,5 casos novos por 100.000 mulheres), ultrapassado apenas pelo câncer de mama. Em Mato Grosso do Sul, essa taxa é de 35,1 por 100.000 , a maior do país e o dobro da média nacional. Ao mesmo tempo, enquanto nos países mais desenvolvidos as taxas de mortalidade por esse câncer vêm apresentando redução, em Mato Grosso do Sul, no período compreendido entre 1979 e 2009, observou-se que as taxas padronizadas pela população mundial passaram de 5,9 para 7,5/100.000 mulheres ${ }^{2}$, o que equivale a um aumento de $26,7 \%$. Três aspectos têm sido apontados como responsáveis pela manutenção das elevadas taxas de mortalidade no país: a cobertura do exame Papanicolaou, seu desempenho e o estadiamento no qual os casos são diagnosticados — todas relacionadas à oportunidade de detectar precocemente a doença por meio do seu rastreamento ${ }^{3}$.

Embora o rastreamento do câncer do colo do útero por meio da citologia cérvico-vaginal seja reconhecido como o método de rastreamento mais bem-sucedido na história da Medicina, sua efetividade está relacionada a uma sequência de eventos que vão desde as estratégias de captação da mulher, coleta do exame citológico, leitura do esfregaço e tratamento oportuno das alterações identificadas ${ }^{4}$. No Brasil, a cobertura da população feminina com exames citológicos periódicos e de qualidade não tem sido suficiente para impactar as elevadas taxas de incidência e mortalidade por câncer de colo do útero ainda observadas em muitas regiões ${ }^{5,6}$. A Organização Pan-Americana da Saúde (OPAS) reforça que a qualidade e a cobertura constituem as maiores deficiências nos programas de prevenção de câncer do colo do útero na América Latina e no Caribe ${ }^{7}$.

Diferentes estratégias têm sido empregadas, com vista à garantia da qualidade dos exames citopatológicos. Bibbo e Wilbur ${ }^{8}$ destacam que além de apresentar problemas com seu financiamento, essas estratégias carecem de objetividade e reprodutibilidade, e não estão livres de vieses. Com essa finalidade, à revisão de lâminas por organizações profissionais ou órgãos governamentais acrescentam-se testes de proficiência por meio da utilização de lâminas previamente validadas, monitoramento do perfil dos laudos emitidos pelo laboratório, correlação cito-histológica, entre outras modalidades. No fim dos anos 1990, laboratórios dos países da América Latina e Caribe foram incentivados a participar da Rede Pan-Americana de Citologia (RedPAC). Duas a três vezes deveriam enviar a um Centro de Referência laudos emitidos individualmente para um conjunto de imagens de citologia cervical e recebiam comentários sobre a concordância entre seus laudos e aqueles fornecidos pelo painel de especialistas. A concordância entre os observadores variou de 0,3 a 0,9 (coeficiente Kappa), sendo que em todos os laboratórios houve aumento nos coeficientes de concordância ${ }^{9}$. A rede chegou a ter a participação de 45 laboratórios localizados no Chile, México, Peru, Costa Rica, Equador, Venezuela e Bolívia?.

No Brasil, a preocupação com a garantia da qualidade dos exames citopatológicos foi oficializada em 1998, quando houve a inclusão do procedimento controle de qualidade do exame citopatológico cérvico-vaginal na tabela de remuneração do Sistema Único de Saúde (SUS) ${ }^{10}$. Tendo por base um estudo pioneiro ocorrido no Paraná entre 1997 e $1999^{11}$, a portaria recomendava a revisão de, pelo menos, $10 \%$ das lâminas por outro observador. Posteriormente, foi estabelecida a obrigatoriedade de participação no processo de monitoramento externo da qualidade (MEQ) dos laboratórios que realizam exames citopatológicos para o SUS ${ }^{12}$. Essa mesma portaria definiu que o MEQ deve ser constituído de uma nova leitura (revisão) da lâmina por um laboratório diferente daquele que realizou a primeira leitura. Como consequência, aos gestores estaduais e municipais foi dada a incumbência de identificar laboratórios para atuar nessas atividades, seguindo as recomendações estabelecidas. O monitoramento externo da qualidade dos esfregaços cérvico-vaginais tem sido uma prática eficiente de educação continuada, permite entender melhor diagnósticos incorretos ou discordantes e planejar estratégias para melhorar o desempenho do laboratório ${ }^{11,12}$.

Recentemente, um conjunto de medidas para fortalecer o Programa Nacional de Controle do Câncer do Colo do Útero e de Mama, lançadas em 22 de março de 2011 pela Presidência da República, incluiu a garantia da qualidade dos exames de rastreamento do SUS entre suas metas.

Este trabalho teve por objetivo analisar a concordância diagnóstica entre os laudos citopatológicos emitidos pelos laboratórios credenciados para atuar na rede SUS do Estado de Mato Grosso do Sul e o Laboratório Central de Saúde Pública do Estado (Lacen).

\section{Métodos}

O Estado de Mato Grosso do Sul contou com 15 laboratórios credenciados para realizar exames citopatológicos para o SUS, entre 2002 e 2011, sendo dez deles na capital (Campo Grande) e cinco no interior (dois em Naviraí e três em Três Lagoas). Como unidade de MEQ foi escolhido o Lacen, que pertence ao Governo do Estado e não está entre os laboratórios credenciados pelo SUS 
para realização dos exames citopatológicos de rotina, garantindo sua isenção no processo. As atividades de MEQ foram iniciadas em outubro de 2001 e desde 2002 atingiram de forma estruturada todos os laboratórios prestadores de serviço do Estado, com registro sistematizado das informações, exceto no ano de 2009, para o qual não há dados disponíveis.

A terminologia empregada para a emissão dos laudos dos exames citológicos foi aperfeiçoada no período compreendido pelo estudo: até abril de 2006 utilizou-se da classificação de Richart para as lesões pré-invasoras associada à conceituação de displasias e carcinoma in situ, assim como de alguns diagnósticos citológicos introduzidos pelo Sistema de Bethesda de 1991, conforme preconizado pelo Ministério da Saúde e Sociedade Brasileira de Citopatologia desde $1993^{13}$. Desde abril de 2006, por recomendação do INCA, passou-se a utilizar a Nomenclatura Brasileira para Laudos Cervicais, uma adaptação do Sistema de Bethesda 2001, que inclui, entre outras alterações, as subdivisões das atipias escamosas/glandulares de significado indeterminado (ASC-US; AGC-US) e não se podendo excluir lesão de alto grau (ASC-H/AGC-H) ${ }^{14}$. A nomenclatura brasileira tem sido revisada constantemente e sua similaridade com o Sistema de Bethesda facilita a equiparação dos resultados nacionais com aqueles encontrados em publicações científicas internacionais.

O Ministério da Saúde ${ }^{14}$ recomenda que sejam encaminhadas para MEQ todas as lâminas com diagnósticos positivos (atipias, lesões intraepiteliais escamosas de baixo e alto grau, carcinomas e adenocarcinomas), todas as lâminas insatisfatórias e uma amostra aleatória dos exames normais até alcançar um total de $10 \%$ de exames selecionados. Essas lâminas são selecionadas mensalmente, de forma automática e aleatória, pelo Sistema de Informação do Câncer do Colo do Útero (SISCOLO), no momento da digitação dos laudos no laboratório onde a lâmina foi examinada. Considerando a capacidade da unidade de MEQ, no Estado de Mato Grosso do Sul foi encaminhada para releitura uma amostra de conveniência daqueles exames que eram selecionados mensalmente pelo SISCOLO para MEQ. Após a releitura dos exames, foram considerados discordantes os casos em que o "novo diagnóstico" implicava mudança de conduta clínica, tendo os resultados sido agrupados em cinco ou quatro categorias, conforme o período analisado: até abril de $2006^{14}$ e a partir de abril de $2006^{15}$, respectivamente (Quadro 1). Foi considerada discordância quando houve mudança de categoria diagnóstica. Fóruns de discussão e revisão das lâminas, com troca de experiência entre os laboratórios participantes, foram desenvolvidos ao longo do período, visando à garantia contínua da qualidade dos exames citopatológicos no Estado.
Quadro 1. Categorias diagnósticas utilizadas para definir discordância entre a primeira e a segunda leituras

\begin{tabular}{|c|c|}
\hline $\begin{array}{l}\text { Nomenclatura até abril de } 2006 \\
\text { ( } 5 \text { categorias) }\end{array}$ & $\begin{array}{l}\text { Nomenclatura a partir de abril de } 2006 \\
\text { (4 categorias) }\end{array}$ \\
\hline Insatisfatória & Insatisfatória para avaliação \\
\hline $\begin{array}{l}\text { Normal } \\
\text { Alterações benignas }\end{array}$ & $\begin{array}{l}\text { Dentro dos limites da normalidade no material } \\
\text { examinado } \\
\text { Alterações celulares benignas }\end{array}$ \\
\hline $\begin{array}{l}\text { Atipia de célula epitelial escamosa de significado } \\
\text { indeterminado (ASCUS) } \\
\text { Atipia de célula glandular de significado } \\
\text { indeterminado (AGUS) } \\
\text { Efeito citopático compatível com HPV (HPV) } \\
\text { Neoplasia intraepitelial cervical grau I (NIC I) }\end{array}$ & $\begin{array}{l}\text { Células escamosas atípicas de significado } \\
\text { indeterminado, possivelmente não neoplásicas } \\
\text { Lesão intraepitelial de baixo grau } \\
\text { Células glandulares atíicas de significado } \\
\text { indeterminado possivelmente não neoplásicas } \\
\text { Células atípicas de origem indefinida } \\
\text { possivelmente não neoplásicas }\end{array}$ \\
\hline $\begin{array}{l}\text { Neoplasia intraepitelial cervical grau II (NIC II) } \\
\text { Neoplasia intraepitelial cervical grau III (NIC III) } \\
\text { Carcinoma invasivo } \\
\text { Adenocarcinoma invasivo } \\
\text { Adenocarcinoma in situ } \\
\text { Outras neoplasias }\end{array}$ & $\begin{array}{l}\text { Células escamosas atíicas de significado } \\
\text { indeterminado, quando não se pode excluir lesão } \\
\text { intraepitelial de alto grau } \\
\text { Lesão intraepitelial de alto grau } \\
\text { Lesão de alto grau não podendo excluir } \\
\text { microinvasão } \\
\text { Carcinoma epidermoide invasor } \\
\text { Células glandulares atipicas de significado } \\
\text { indeterminado em que não se pode excluir lesão } \\
\text { intraepitelial de alto grau } \\
\text { Adenocarcinoma in situ } \\
\text { Adenocarcinoma invasor } \\
\text { Células atíicas de origem indefinida } \\
\text { possivelmente não neoplásicas em que não se } \\
\text { pode afastar lesão de alto grau } \\
\text { Outras neoplasias }\end{array}$ \\
\hline
\end{tabular}

Relatórios sintéticos de monitoramento externo da qualidade dos exames citológicos emitidos pelo SISCOLO foram transcritos para um aplicativo construído em planilha Excel (Microsoft Corporation, versão 5.1, 2002) conforme orientações do INCA ${ }^{16}$, sendo calculados automaticamente o coeficiente de concordância global e o coeficiente Kappa de Cohen. O coeficiente de concordância global e o coeficiente Kappa de Cohen foram calculados a partir das seguintes fórmulas:

Coeficiente de número total de acordos x 100 concordância global $=$ e total de pares de diagnósticos Kappa de Cohen $=\frac{\text { concordância esperada ao acaso }}{1-\text { concordância esperada ao acaso }}$ proporção de concordantes -

Para os 13 laboratórios com informações disponíveis para dois ou mais anos a comparação entre os valores de concordância dos anos inicial e final foi computada por meio da variação absoluta $(\operatorname{delta}(\Delta)=$ coeficiente final - coeficiente inicial) e variação percentual relativa $(\mathrm{VPR}=$ [coeficiente final - coeficiente inicial $* 100$ / coeficiente inicial). Os outros dois laboratórios, submetidos a MEQ em apenas um ano, foram excluídos dessa análise. Os resultados do 
coeficiente Kappa foram classificados conforme o proposto por Byrt ${ }^{17} \mathrm{em}$ : concordância perfeita $(\kappa=1,0)$, concordância excelente $(\kappa>0,8)$, concordância boa $(0,6<\kappa<0,8)$, concordância moderada $(0,4<\kappa<0,6)$, concordância pobre $(0<\kappa<0,4)$ e ausência de concordância $(\kappa=0)$.

Por se tratar de pesquisa baseada em dados administrativos, unicamente na comparação de laudos, não foi necessária submissão a um Comitê de Ética em Pesquisa.

\section{Resultados}

Entre os anos de 2002 a 2011 foram encaminhadas para releitura 15.989 esfregaços, referentes a 15 laboratórios, perfazendo 62 remessas de exames. O número de lâminas analisadas, por ano, por laboratório, variou de 1 a 1.372, conforme a disponibilidade do Lacen e a seleção mensal gerada automaticamente pelo SISCOLO.

Tabela 1. Distribuição percentual dos resultados iniciais dos exames citopatológicos encaminhados para MEQ. Mato Grosso do Sul, no período de 2002 a 2011

\begin{tabular}{lcc}
\hline \multirow{2}{*}{ Categoria diagnóstica } & \multicolumn{2}{c}{ Exames encaminhados } \\
\cline { 2 - 3 } & $\mathrm{n}$ & $\%$ \\
\hline Normal / alteração benigna & 7.696 & 48,1 \\
Atipia / lesão de baixo grau & 5.800 & 36,3 \\
Lesão de allo grau / Carcinoma / & 676 & 4,2 \\
Adenocarcinoma & & \\
Insatisfatório & 1.817 & 11,4 \\
Total & 15.989 & 100,0 \\
\hline
\end{tabular}

Tabela 2. Valores mediano, mínimo e máximo do coeficiente de concordância global e do coeficiente Kappa de Cohen conforme 0 ano analisado

\begin{tabular}{|c|c|c|c|}
\hline Estatística & Valor mediano & Valor mínimo & Valor máximo \\
\hline \multicolumn{4}{|c|}{ Coeficiente de concordância global } \\
\hline 2002 & 0,7 & 0,2 & 0,8 \\
\hline 2003 & 0,9 & 0,8 & 1,0 \\
\hline 2004 & 0,8 & 0,5 & 1,0 \\
\hline 2005 & 1,0 & 0,7 & 1,0 \\
\hline 2006 & 1,0 & 1,0 & 1,0 \\
\hline 2007 & 0,9 & 0,6 & 1,0 \\
\hline 2008 & 1,0 & 0 & 1,0 \\
\hline 2009 & ND & ND & ND \\
\hline 2010 & 0,9 & 0,6 & 1,0 \\
\hline 2011 & 1,0 & 1,0 & 1,0 \\
\hline \multicolumn{4}{|c|}{ Coeficiente Kappa } \\
\hline 2002 & 0,5 & 0,1 & 0,7 \\
\hline 2003 & 0,8 & 0,5 & 0,9 \\
\hline 2004 & 0,8 & 0,3 & 1,0 \\
\hline 2005 & 0,9 & 0,5 & 1,0 \\
\hline 2006 & 0,9 & 0,9 & 0,9 \\
\hline 2007 & 0,8 & 0,3 & 0,9 \\
\hline 2008 & 0,9 & 0 & 0,9 \\
\hline 2009 & ND & ND & ND \\
\hline 2010 & 0,9 & 0,3 & 1,0 \\
\hline 2011 & 0,9 & 0,8 & 1,0 \\
\hline
\end{tabular}

ND: Dados não disponíveis
Observou-se que, na primeira avaliação, a maior parte dos esfregaços apresentava-se normal ou com alteração benigna $(48,1 \%)$, seguidos de diagnóstico de atipia ou lesão de baixo grau (36,3\%). Pouco mais de $11 \%$ das lâminas foram consideradas insatisfatórias para leitura (Tabela 1).

O coeficiente de concordância global (Tabela 2) oscilou entre 0,2 e 1,0 , sendo que o valor mediano passou de 0,7 em 2002 para 1,0 em $2011(\mathrm{VPR}=+36,6 \%)$. A análise dos valores do coeficiente Kappa mostrou três casos em que a concordância foi perfeita (4,8\%), 29 em que a concordância foi excelente (46,8\%), 12 em que a concordância foi boa $(19,4 \%)$ e 6 em que foi classificada como pobre $(9,7 \%)$ - enquanto em 2002 nenhum laboratório apresentou concordância perfeita ou excelente (coeficiente Kappa >0,80). Em 2011, dos oito laboratórios avaliados, sete atingiram essa classificação, havendo apenas um cuja concordância foi considerada boa (dados não mostrados). Os valores medianos do coeficiente Kappa aumentaram de 0,5 em 2002 para 0,9 em 2011, representando um aumento de mais de $80 \%(\mathrm{VPR}=+80,8 \%)$. Em um único caso houve piora no coeficiente Kappa $(\Delta=-25 \%)$; Os outros 12 laboratórios apresentaram melhoras no coeficiente Kappa que variaram de $+12 \mathrm{a}+91$ pontos percentuais (valor mediano de $\Delta=+32 \%$ ).

\section{Discussão}

Neste estudo foi analisada a concordância diagnóstica entre 15.989 laudos citopatológicos emitidos pelos 15 laboratórios credenciados pela Secretaria Estadual de Saúde de Mato Grosso do Sul para atuar no SUS entre 2002 e 2011 e a unidade de MEQ. Em primeiro lugar é importante destacar que se trata de atividade relativamente recente no país, somente incorporada ao SUS a partir de 1998, por ocasião da estandardização pelo Viva Mulher - Programa Nacional de Controle do Câncer do Colo do Útero, das ações nacionais de controle do câncer do colo do útero. Nesse cenário, os resultados apresentados mostraram a exequibilidade do MEQ dos exames citopatológicos na esfera estadual, sua sustentabilidade ao longo de 10 anos e a contribuição para a garantia da qualidade dos diagnósticos feitos no SUS, demonstrada pela crescente melhoria nos índices de concordância obtidos.

Além da diversidade de métodos utilizados em outros países para MEQ dos exames citopatológicos, a crescente automação dos exames, a utilização de citologia em meio líquido, a implementação de ações de monitoramento interno da qualidade (que minimiza a necessidade do MEQ) e a peculiaridade do modelo de MEQ adotado no Brasil tornam difícil a comparação dos resultados obtidos com os de outros estudos publicados. Dessa forma, os autores optaram por comparar os resultados de Mato Grosso do Sul aos de outras experiências nacionais bem-sucedidas publicadas. 
Após a revisão de 65.753 citologias cérvico-vaginais provenientes dos 52 laboratórios participantes do Programa de Prevenção do Câncer Ginecológico do Estado do Paraná ${ }^{11}$, entre outubro de 1997 e julho de 1999, observou-se coeficiente de concordância de $97,0 \%$ e coeficiente Kappa de $88,8 \%$ entre a leitura original e a releitura das lâminas. Os autores concluíram que a releitura de $10 \%$ das lâminas mostrou-se efetiva para monitoramento externo da qualidade em regiões de baixa renda. Nesse estudo, os revisores conheciam o laudo inicial, o que pode ter influenciado as análises.

Já em São Paulo, o Instituto Adolfo Lutz e a Fundação Oncocentro (dados não publicados), no período de 2000 a 2009, monitoraram a qualidade dos laboratórios prestadores de serviço ao SUS no Estado por meio da revisão de 115.265 lâminas aplicando a mesma metodologia. O coeficiente de concordância global notado $(86,9 \%)$ foi inferior ao descrito na maior parte dos anos em Mato Grosso do Sul: somente nos três primeiros anos (2002 a 2004) foram observados valores inferiores aos de São Paulo. Entretanto, cabe ressaltar que, em São Paulo a análise foi feita com base em 7 categorias: insatisfatório, negativo, atipias-US, atipias H, LSIL, HSIL e lesões invasivas, o que pode justificar a diferença nos resultados. Os autores destacam a importante redução no percentual de resultados falso-positivos (de 1,5 para $0,2 \%)$. Em publicação sobre período anterior ${ }^{18}$ referente aos exames monitorados entre 2000 e 2004 , o mesmo grupo havia observado coeficiente de concordância global de $85,8 \%$, com redução de discordância nas categorias LSIL, HSIL e lesões invasivas, o que se manteve na publicação mais recente. O coeficiente Kappa variou de 0,7 (ano 2000) a 0,8 (ano 2004).

Por outro lado, um estudo piloto ocorrido em Goiânia analisou 537 esfregaços selecionados pelo SISCOLO durante o ano de 2003, obtendo-se coeficiente de concordância global de 98,9\% nas 9 categorias diagnósticas analisadas ${ }^{19}$. Conforme destacam Collaço et al. ${ }^{11}$, particularidades metodológicas dos estudos analisados e a experiência dos profissionais envolvidos podem explicar as diferenças observadas nos resultados.

Os percentuais de lâminas com lesão de alto grau, carcinoma ou adenocarcinoma na leitura inicial registrados em Curitiba $(3,5 \%)^{11}$ e São Paulo (3,8\% - dados não publicados) foram inferiores aos $4,2 \%$ observados no presente estudo, o que está em sintonia com as taxas de incidência de câncer do colo do útero mais elevadas projetadas para Mato Grosso do Sul em 2012 (35,1 por 100.000 mulheres versus 13,8 para o Estado de São Paulo e 13,2 para o Estado do Paraná $\left.{ }^{1}\right)$. Por sua vez, em Goiânia $^{19} 7,7 \%$ dos laudos iniciais referiam-se a essas categorias diagnósticas, coerente com a elevada taxa de incidência estimada para essa cidade em 2012: 24,3 por 100.000 mulheres.

Além disso, comparativamente a Mato Grosso do Sul $(11,4 \%)$ o percentual de exames insatisfatórios encaminhado para MEQ foi quase duas vezes superior em Goiânia $(21,0 \%)^{19}$, mas menos da metade em São Paulo $\left(5,1 \%\right.$ - dado não publicado) e no Paraná $(1,8 \%)^{11}$, o que pode estar associado, entre outras coisas, à antiguidade e eficácia do programa de monitoramento, aos programas de educação continuada desenvolvidos e às políticas locais para credenciamento e descredenciamento dos laboratórios nesses estados. É importante salientar que esse percentual de exames insatisfatórios encaminhados para MEQ é função das regras de seleção do SISCOLO e da logística implementada pelo gestor local.

A partir dos resultados obtidos pode-se concluir pela exequibilidade do MEQ dos exames citopatológicos na esfera estadual e que sua implementação resulta em melhoria nos diagnósticos realizados na rede SUS.

\section{Agradecimentos}

Aos profissionais dos laboratórios de citopatologia, por suas importantes contribuições para implantação das atividades de MEQ no Estado de Mato Grosso do Sul.

\section{Referências}

1. Brasil. Ministério da Saúde. Instituto Nacional de Câncer. Estimativa 2012: incidência de câncer no Brasil. Rio de Janeiro: INCA; 2011.

2. Brasil. Ministério da Saúde. Instituto Nacional de Câncer [Internet]. Atlas de mortalidade por câncer. Rio de Janeiro: INCA; 2008 [cited $2010 \mathrm{Jul}$ 5]. Available from: http://mortalidade.inca.gov. br/Mortalidade/

3. Thuler LCS. Mortality due to cancer of the uterine cervix in Brazil. Rev Bras Ginecol Obstet. 2008;30(5):216-8.

4. Thuler LCS, Zardo LM, Zeferino LC. Perfil dos laboratórios de citopatologia do sistema único de saúde. J Bras Patol Med Lab. 2007;43(2):106-14.
5. Ozawa C, Marcopito LF. Papanicolaou smear screening: coverage in two home surveys applied in the city of São Paulo in 1987 and 2001-2002. Rev Bras Ginecol Obstet. 2011; $33(5): 238-45$.

6. Freitas HG, Silva MA, Thuler LCS. Câncer do colo do útero no Estado de Mato Grosso do Sul: ações de detecção precoce, incidência e mortalidade. Rev Bras Cancerol. No prelo 2012;58(3).

7. Lewis M. A situational analysis of cervical cancer in Latin America and the Caribbean. Washington (DC): PAHO; 2004.

8. Bibbo M, Wilbur D. Comprehensive cytopathology, expert consult: online and print. 3rd ed. Philadelphia: Saunders; 2008. 
9. Murillo R, Almonte M, Pereira A, Ferrer E, Gamboa OA, Jerónimo $\mathrm{J}$, et al. Cervical cancer screening programs in Latin America and the Caribbean. Vaccine. 2008;26(Suppl 11):L37-48.

10. Brasil. Ministério da Saúde. Secretaria de Assistência à Saúde. Portaria n. 79, 6 julho 1998. Diário Oficial da República Federativa do Brasil, Brasília (DF) 1998 Jul 8.

11. Collaço LM, de Noronha L, Pinheiro DL, Bleggi-Torres LF. Quality assurance in cervical screening of a high risk population: a study of 65,753 reviewed cases in Parana Screening Program, Brazil. Diagn Cytopathol. 2005;33(6):441-8.

12. Brasil. Ministério da Saúde. Secretaria de Políticas de Saúde. Secretaria de Assistência à Saúde. Portaria conjunta n. 92, 16 outubro 2001. Diário Oficial da República Federativa do Brasil, Brasília (DF) 2001 Out 17.

13. Seminário nacional sobre nomenclatura e controle de qualidade dos exames citológicos e prevenção do câncer cérvico-uterino; 1993 Out 5-6. Rio de Janeiro: INCA; 1993.

14. Brasil. Ministério da Saúde. Instituto Nacional de Câncer. Nomenclatura brasileira para laudos citopatológicos cervicais e condutas clínicas preconizadas. Rio de Janeiro: INCA; 2003.
15. Brasil. Ministério da Saúde. Secretaria de Atenção à Saúde. Instituto Nacional de Câncer. Coordenação de Prevenção e Vigilância. Nomenclatura brasileira para laudos cervicais e condutas preconizadas: recomendações para profissionais de saúde. 2a ed. Rio de Janeiro: INCA; 2006.

16. Brasil. Ministério da Saúde. Instituto Nacional de Câncer. Monitoramento externo da qualidade do exame citopatológico: procedimentos para avaliação da concordância entre laboratórios de citopatologia. Rio de Janeiro: INCA; 2002.

17. Byrt T. How good is that agreement? Epidemiology. 1996;7(5):561.

18. Pereira SMM, Ramos DEL, Yamamoto LSU, Shirata NK, di Loreto C, Ferraz MGMC, et al. Monitoramento externo de qualidade em citopatologia cervical e o reflexo na rotina dos laboratórios da rede pública. DST J Bras Doenças Sex Transm. 2006; 18(3): 172-7.

19. Amaral RG, Souza NLA, Tavares SBN, Manrique EJC, Assem DZ, Azevedo $L$, et al. Controle externo da qualidade dos diagnósticos citológicos no rastreamento do câncer cervical: estudo piloto. RBAC. 2006;38(2):79-81. 